\title{
Renal cell carcinoma with non-clear cell histology or sarcomatoid differentiation: recent insight in an unmet clinical need
}

\author{
Frede Donskov \\ Department of Oncology, Aarhus University Hospital, Aarhus, Denmark \\ Correspondence to: Professor Frede Donskov, MD, DMSc. Department of Oncology, Aarhus University Hospital, Palle Juul-Jensens Boulevard 99, \\ 8200 Aarhus N, Denmark. Email: frede.donskov@auh.rm.dk. \\ Comment on: McGregor BA, McKay RR, Braun DA, et al. Results of a Multicenter Phase II Study of Atezolizumab and Bevacizumab for Patients \\ With Metastatic Renal Cell Carcinoma With Variant Histology and/or Sarcomatoid Features. J Clin Oncol 2020;38:63-70. \\ Tannir NM, Signoretti S, Choueiri TK, et al. Efficacy and Safety of Nivolumab Plus Ipilimumab versus Sunitinib in First-line Treatment of Patients \\ with Advanced Sarcomatoid Renal Cell Carcinoma. Clin Cancer Res 2021;27:78-86. \\ Rini BI, Motzer RJ, Powles T, et al. Atezolizumab plus Bevacizumab Versus Sunitinib for Patients with Untreated Metastatic Renal Cell Carcinoma \\ and Sarcomatoid Features: A Prespecified Subgroup Analysis of the IMmotion151 Clinical Trial. Eur Urol 2020. [Epub ahead of print]. doi: 10.1016/ \\ j.eururo.2020.06.021.
}

Submitted Oct 20, 2020. Accepted for publication Dec 11, 2020.

doi: $10.21037 /$ atm-20-7009

View this article at: http://dx.doi.org/10.21037/atm-20-7009

Renal cell carcinoma (RCC) is the 13 th most common malignancy resulting in more than 400,000 new cases and 175,000 deaths per year worldwide (1). The histologic subset clear cell RCC (ccRCC), or conventional RCC, comprises $75 \%$ of cases. Clinical trials for 20 years in patients with metastatic RCC (mRCC) have therefore focused on patients with clear cell histology. Today 15 drugs have shown clinical benefit in this group of patients (Figure 1), leading to substantial, clinically meaningful improved outcomes for the vast majority of patients with mRCC (2). A wide availability of diagnostic CT has led to stage migration, with more than half of patients with RCC now identified as incidental findings (3). For patients with localized RCC, surgical options have improved with minimally invasive techniques, including robotical techniques and laparoscopic techniques (4), and renal cryoablation has also shown promise (5). Moreover, decline in tobacco use and increased physical activity (6) contribute to the improved results. In essence, mortality from RCC is declining (7); a recent nationwide study showed a $7 \%$ yearly reduction in mortality from RCC (8). With this achievement in the vast majority of patients, focus has shifted to the remaining RCC patients with area of needs. Progress has now been made in patients with non-clear cell histology (non-ccRCC), also called rare histologic variants, and patients with sarcomatoid histology.

Non-ccRCC comprises $25 \%$ of RCC and is a diverse group of tumors that includes papillary type I, papillary type II, chromophobe, medullary, collecting duct, TFE3 translocation, and unclassified RCC. Outcomes with targeted agents have generally been poor in patients with non-ccRCC (9-12). The ASPEN and ESPN trials, evaluating everolimus versus sunitinib, showed modest efficacy, with objective response rates (ORR) less than $20 \%$, including less than $1 \%$ complete response (CR), and median progression free survival (PFS) less than 9 months $(13,14)$. Interleukin-2 based immunotherapy did not show efficacy in non-ccRCC (15). In addition, both ccRCC and non-ccRCC may be associated with sarcomatoid differentiation (sarcomatoid RCC, sRCC). Only 10-20\% of patients with mRCC have sRCC, but sRCC is associated with an aggressive phenotype, and an advanced stage at diagnosis (16); the rate of sarcomatoid differentiation was doubled in synchronous versus metachronous mRCC (17). These patients have a particularly poor prognosis, and systemic therapies in sRCC have been mostly ineffective $(18,19)$.

In 2020/2021, three important studies have brought about greater insight in this specific area. Bradley A. McGregor and colleagues reported in the January 2020 edition of Fournal of Clinical Oncology the results of an investigator-initiated multicenter Phase II study of atezolizumab and bevacizumab for patients with metastatic RCC with non-ccRCC and/or sarcomatoid features (20). The study included a total of 60 patients 


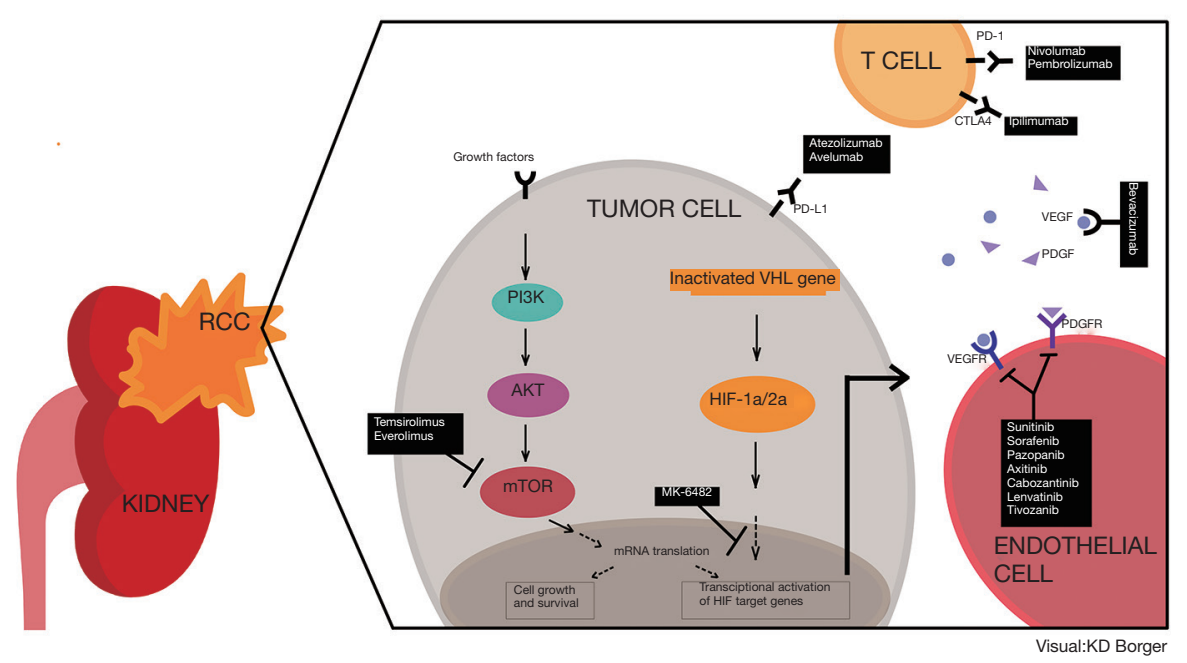

Figure 1 Systemic treatment options in metastatic renal cell carcinoma. RCC, renal cell carcinoma; VHL, von Hippel-Lindau; HIF, Hypoxia-inducible factor; VEGF, Vascular endothelial growth factor; VEGFR, Vascular endothelial growth factor receptor; PDGF, Plateletderided growth factor; PDGFR, Platelet-derided growth factor receptor, PD-1, Programmed cell death protein-1; PD-L1, Programmed death ligand-1; CTLA-4, cytotoxic T-lymphocyte-associated protein-4.

at 4 academic institutions in the USA; 42 patients (70\%) with non-ccRCC and 18 (30\%) with ccRCC with $\geq 20 \%$ sarcomatoid differentiation. Most patients had prior nephrectomy, i.e., had metachronous metastatic disease (17) and the presence of bone metastases was unusually low $(2 \%)$, therefore, selection of patients with less aggressive underlying biology may be suspected. The primary endpoint was ORR as the best overall response by RECIST version 1.1, by investigator assessment. Patients were treated with atezolizumab $1,200 \mathrm{mg}$ and bevacizumab $15 \mathrm{mg} / \mathrm{kg}$ intravenously every 3 weeks. Median time of follow-up was 13 months. The ORR for the overall population was $33 \%$, with 2 patients (3\%) obtaining a CR, $50 \%$ of patients with ccRCC with sarcomatoid differentiation, and $26 \%$ in patients with non-ccRCC. The overall median PFS was 8.3 months (95\% CI, 5.7 to 10.9 months), median OS was not reached; the PFS/OS results for subgroups with sarcomatoid or non-ccRCC was not provided. A baseline biopsy was mandatory per protocol. Tissue was, however, not suitable for PD-L1 staining, therefore, archival nephrectomy tissue was used instead in $36(60 \%)$ patients. The authors used a PD-L1 score $\geq 1 \%$ tumor cells to determine positivity. Overall, $42 \%$ of patients were PD-L1 positive. Overall, ORR in PDL1positive vs. PD-L1-negative patients was $60 \%$ vs. $19 \%$; in ccRCC with sarcomatoid differentiation $50 \%$ vs. $29 \%$, respectively; in patients with non-ccRCC $67 \%$ vs. $14 \%$. A summary of key findings is listed in Table 1 .

IMmotion151 was a randomized, open-label, phase III study evaluating the efficacy and safety of atezolizumab plus bevacizumab versus sunitinib in patients with previously untreated, inoperable RCC with a clear cell component (23). A prespecified subgroup analysis of patients with sRCC was recently published by Brian Rini and colleagues in Eur Urol (21). Of the 915 intention-to-treat (ITT) patients included in IMmotion151, a total of $142(15.5 \%)$ patients with sRCC (81\% had ccRCC, 19\% had non-ccRCC) received atezolizumab + bevacizumab or sunitinib. Moreover, PD-L1 expression $(\geq 1 \%$ vs. $<1 \%)$ on tumor-infiltrating immune cells was assessed. In total, 86 patients $(9.4 \%$ of ITT) had both sarcomatoid features as well as being PD-L1 positive. The median follow-up was 13 months. In patients with sarcomatoid features, results showed improved outcomes with atezolizumab plus bevacizumab versus sunitinib; median PFS 8.3 vs. 5.3 months (HR 0.52); median OS 21.7 vs. 15.4 months (HR 0.64); ORR 49\% vs. $14 \%$ with CR rate $10 \%$ vs. $3 \%$ and ongoing response in $52 \%$ vs. $30 \%$ (Table 1). In the subset of patients with both sarcomatoid features and PD-L1 positive staining almost identical results was noted; median PFS 8.6 vs. 5.6 months (HR 0.45); median OS 19.3 vs. 15.0 months (HR 0.61); ORR $56 \%$ vs. $12 \%$ with CR rate $14 \%$ vs. $4 \%$ and ongoing response in $45 \%$ vs. $0 \%$. Data in patients with both sarcomatoid features and PD-L1 negative staining was not provided. However, compared with 
Table 1 Key study findings in patients with metastatic renal cell carcinoma with sarcomatoid differentiation and/or non-clear cell histology

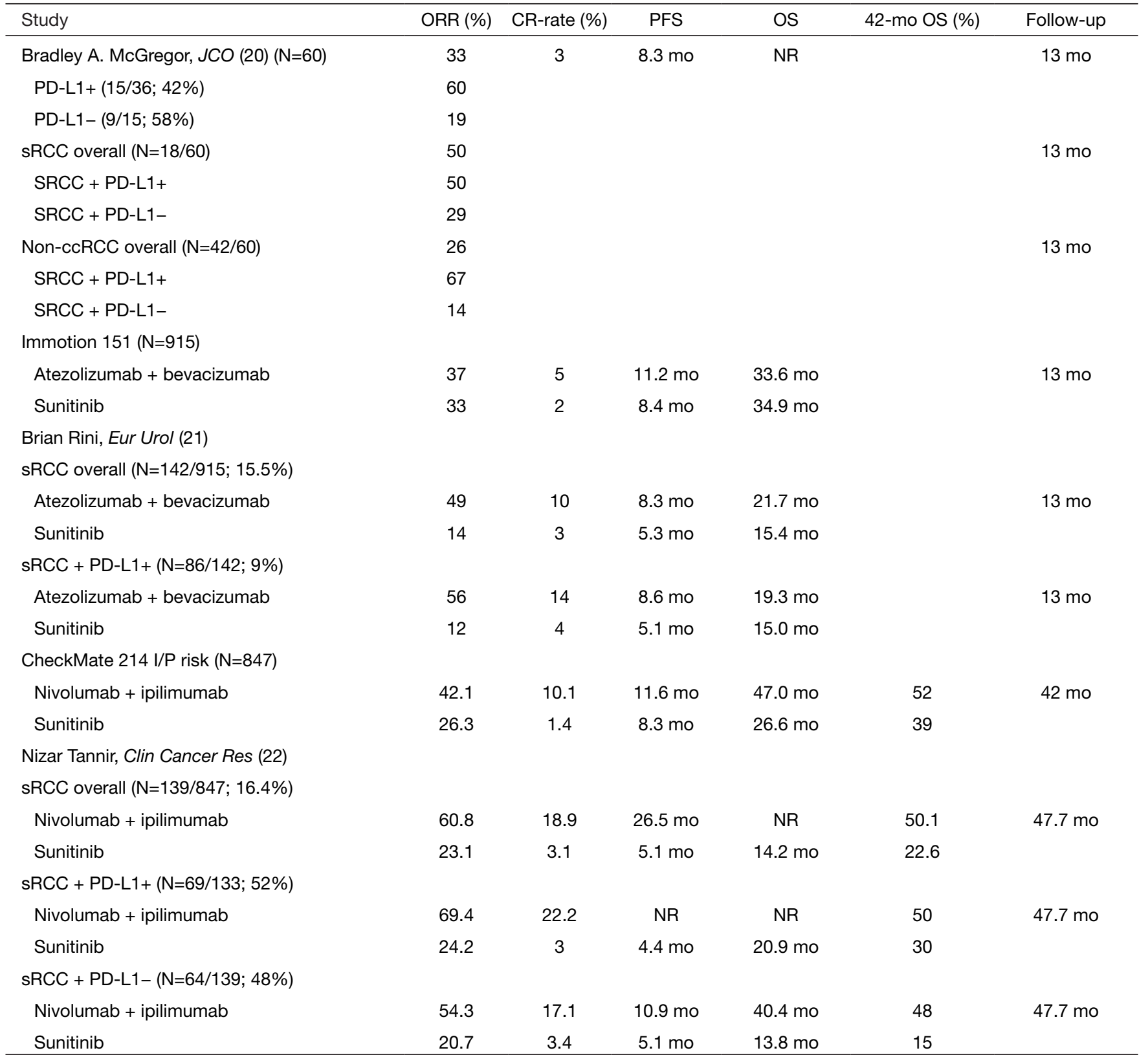

sRCC, sarcomatoid renal cell carcinoma; ccRCC, clear cell renal cell carcinoma; ORR, objective response rate; CR, complete response; PFS, median progression free survival; OS, median overall survival.

the intention to treat population where patients treated with atezolizumab plus bevacizumab had PFS of 11.2 months and OS of 33.6 months, outcomes for patients with sarcomatoid features or sarcomatoid features with PD-L1 expression were less favorable, and treatment with atezolizumab plus bevacizumab did not overcome the negative prognostic impact of sarcomatoid differentiation or PD-L1 expression.
CheckMate 214 was a randomized phase III study evaluating the efficacy and safety of nivolumab plus ipilimumab versus sunitinib (24). A post hoc analysis in patients with sRCC was recently published by Nizar Tannir and colleagues in Clinical Cancer Research (22). Of the 1,096 patients included in the phase III study, 139 (13\%) patients had sRCC and were IMDC intermediate/ 
poor-risk; the ratio of sRCC with ccRCC $v s$. non-ccRCC histology was not given. The baseline tumor cell PDL1 expression was assessed and approximately half of patients had a positive tumor PD-L1 expression. Minimum follow-up was 47.7 months. Nivolumab plus ipilimumab showed notable benefits over sunitinib in patients with sRCC; median OS not reached vs. 14.2 months (HR $0.45)$; 42 -month probability of survival $50.1 \%$ vs. $22.6 \%$; median PFS 26.5 vs. 5.1 months (HR 0.54); 36-month probability of PFS $48.2 \%$ vs. $20.3 \%$; ORR $60.8 \%$ vs. $23.1 \%$, with CR-rate of $18.9 \%$ vs. $3.1 \%$, respectively (Table 1). Moreover, ongoing response was observed in $69 \%$ vs. $53 \%$. Efficacy outcomes were notably better with nivolumab plus ipilimumab versus sunitinib, regardless of PD-L1 expression; $22.2 \%$ of patients with sRCC and tumor PD-L1 expression $\geq 1 \%$ achieved CR with NIVO+IPI, and $17.1 \%$ of patients with sRCC and tumor PD-L1 expression $<1 \%$ achieved CR with NIVO+IPI.

The data presented here indicates with a 42 months' minimum follow-up, that nivolumab plus ipilimumab offers patients with IMDC intermediate/poor risk and sRCC the potential of ORR in two thirds of cases; CR in approximately one fifth; durable responses in two thirds of cases obtaining response; 3-year PFS-plateau of 48\%; identical OS-plateau in sRCC as in non-sRCC patients; and identical OS-plateau in sRCC patients regardless of PD-L1 expression. This supports nivolumab plus ipilimumab as a standard of care for first-line treatment in patients with sRCC disease. In essence, the negative prognostic impact of sRCC as well as PD-L1 expression was overcome with the use of nivolumab plus ipilimumab. We are awaiting long-term follow-up data from each of the three trials; KEYNOTE-426 trial assessing pembrolizumab plus axitinib versus sunitinib, JAVELIN Renal 101 trial assessing avelumab plus axitinib versus sunitinib, and CheckMate 9ER assessing nivolumab plus cabozantinib versus sunitinib.

Sarcomatoid transformation in RCC is characterized by a transformative growth pattern of the epithelial neoplasm into malignant spindle-shaped cells. Histologically, sRCC contains both epithelial (carcinomatous) and mesenchymal (sarcomatoid) components, unlike a true sarcoma of the kidney. The correct diagnosis of sRCC is based on the experience of the pathologist and is based on the degree of assessment. Assessment of a core biopsy versus thorough assessment of the entire nephrectomy specimen may impact findings. Also the timepoint of assessment has impact; assessment at the time of metastasis or at the time of primary diagnosis will impact the rate of sRCC diagnosis.
Finally, is the correct cutoff $20 \%$ or $1 \%$ ? These dilemmas were all clearly illustrated by the articles reviewed (20-22). In most cases, an underestimation of sRCC may occur. Nevertheless, whereas sRCC used to be a marker of severe poor prognosis and resistant to therapy, treatment with nivolumab and ipilimumab now offers hope of a potential cure in a substantial subset of patients with sRCC. This must be considered a truly remarkable and monumental achievement within the research of sRCC.

PD-L1 stating is without consistency in mRCC, as illustrated by the above studies. Three different antibodies were used in the three studies: 405.9A11 mouse monoclonal antibody (20), VENTANA PD-L1 SP142 assay (21), and Dako PD-L1 IHC 28-8 pharmDx test (22), while staining was on either tumor cells $(20,22)$ or tumor-infiltrating immune cells (21). This highly illustrates the lack of consistency in assessment methods. PD-L1 expression as a predictive marker has been assessed for a decennium. In patients with mRCC this marker only has a role as a negative prognostic factor, not a predictive factor for therapy selection, as both patients with PD-L1 positive as well as PDL1 negative tumors benefit from therapy. Treatment with nivolumab in combination with ipilimumab now represents the new backbone of therapy for patients with IMDC intermediate/poor risk or sRCC, irrespective of PD-L1 expression. Moreover, for patients with sRCC, the negative prognostic impact of sRCC as well as PD-L1 expression were overcome with use of nivolumab plus ipilimumab.

Other areas of need for patients with mRCC may now hopefully be the focus of further intense investigations, especially patients with brain metastasis. Other areas of need include: patients with poor performance status, patients with synchronous metastatic disease, patients with considerable co-morbidity, patients with rapidly growing tumors and concomitant symptoms, and patients with bone metastases or lymph node metastases. Therefore, clinical trials within the aforementioned areas are greatly welcomed.

\section{Acknowledgments}

Funding: FD received research grant from the Health Research Fund Central Denmark Region.

\section{Footnote}

Provenance and Peer Review: This article was commissioned by the editorial office, Annals of Translational Medicine. The article did not undergo external peer review. 
Conflicts of Interest: The author has completed the ICMJE uniform disclosure form (available at http://dx.doi. org/10.21037/atm-20-7009). Dr. FD reports grants from Pfizer, from Ipsen, from MSD, outside the submitted work.

Ethical Statement: The author is accountable for all aspects of the work in ensuring that questions related to the accuracy or integrity of any part of the work are appropriately investigated and resolved.

Open Access Statement: This is an Open Access article distributed in accordance with the Creative Commons Attribution-NonCommercial-NoDerivs 4.0 International License (CC BY-NC-ND 4.0), which permits the non-commercial replication and distribution of the article with the strict proviso that no changes or edits are made and the original work is properly cited (including links to both the formal publication through the relevant DOI and the license). See: https://creativecommons.org/licenses/by-nc-nd/4.0/.

\section{References}

1. Bray F, Ferlay J, Soerjomataram I, et al. Global cancer statistics 2018: GLOBOCAN estimates of incidence and mortality worldwide for 36 cancers in 185 countries. CA Cancer J Clin 2018;68:394-424.

2. Chowdhury N, Drake CG. Kidney Cancer: An Overview of Current Therapeutic Approaches. Urol Clin North Am 2020;47:419-31.

3. Ljungberg B, Albiges L, Abu-Ghanem Y, et al. European Association of Urology Guidelines on Renal Cell Carcinoma: The 2019 Update. Eur Urol 2019;75:799-810.

4. Li M, Cheng L, Zhang H, et al. Laparoscopic and Robotic-Assisted Partial Nephrectomy: An Overview of Hot Issues. Urol Int 2020;104:669-77.

5. Nielsen TK, Vedel PF, Borgbjerg J, et al. Renal cryoablation: five- and 10-year survival outcomes in patients with biopsy-proven renal cell carcinoma. Scand J Urol 2020. doi: 10.1080/21681805.2020.1794954.

6. Matthews CE, Moore SC, Arem H, et al. Amount and Intensity of Leisure-Time Physical Activity and Lower Cancer Risk. J Clin Oncol 2020;38:686-97.

7. Levi F, Ferlay J, Galeone C, et al. The changing pattern of kidney cancer incidence and mortality in Europe. BJU Int 2008;101:949-58.

8. Danckert B, Horsbøl TA, Andersen O, et al. Registrations of Patients with Renal Cell Carcinoma in the Nationwide Danish Renal Cancer Database versus the Danish Cancer
Registry: Data Quality, Completeness and Survival (DaRenCa Study-3). Clin Epidemiol 2020;12:807-14.

9. Kroeger N, Xie W, Lee JL, et al. Metastatic non-clear cell renal cell carcinoma treated with targeted therapy agents: characterization of survival outcome and application of the International mRCC Database Consortium criteria. Cancer 2013;119:2999-3006.

10. Harshman LC, Kroeger N, Rha SY, et al. First-line Mammalian target of rapamycin inhibition in metastatic renal cell carcinoma: an analysis of practice patterns from the International Metastatic Renal Cell Carcinoma Database Consortium. Clin Genitourin Cancer 2014;12:335-40.

11. Yip SM, Ruiz Morales JM, Donskov F, et al. Outcomes of Metastatic Chromophobe Renal Cell Carcinoma (chrRCC) in the Targeted Therapy Era: Results from the International Metastatic Renal Cell Cancer Database Consortium (IMDC). Kidney Cancer 2017;1:41-7.

12. Connor Wells J, Donskov F, Fraccon AP, et al. Characterizing the outcomes of metastatic papillary renal cell carcinoma. Cancer Med 2017;6:902-9.

13. Armstrong AJ, Halabi S, Eisen T, et al. Everolimus versus sunitinib for patients with metastatic non-clear cell renal cell carcinoma (ASPEN): a multicentre, open-label, randomised phase 2 trial. Lancet Oncol 2016;17:378-88.

14. Tannir NM, Jonasch E, Albiges L, et al. Everolimus Versus Sunitinib Prospective Evaluation in Metastatic Non-Clear Cell Renal Cell Carcinoma (ESPN): A Randomized Multicenter Phase 2 Trial. Eur Urol 2016;69:866-74.

15. Upton MP, Parker RA, Youmans A, et al. Histologic predictors of renal cell carcinoma response to interleukin-2-based therapy. J Immunother 2005;28:488-95.

16. Zhang $\mathrm{L}, \mathrm{Wu} \mathrm{B}, \mathrm{Zha} \mathrm{Z}$, et al. The prognostic value and clinicopathological features of sarcomatoid differentiation in patients with renal cell carcinoma: a systematic review and meta-analysis. Cancer Manag Res 2018;10:1687-703.

17. Donskov F, Xie W, Overby A, et al. Synchronous Versus Metachronous Metastatic Disease: Impact of Time to Metastasis on Patient Outcome-Results from the International Metastatic Renal Cell Carcinoma Database Consortium. Eur Urol Oncol 2020;3:530-9.

18. Mouallem NE, Smith SC, Paul AK. Sarcomatoid renal cell carcinoma: Biology and treatment advances. Urol Oncol 2018;36:265-71.

19. Kyriakopoulos CE, Chittoria N, Choueiri TK, et al. Outcome of patients with metastatic sarcomatoid renal cell 
carcinoma: results from the International Metastatic Renal Cell Carcinoma Database Consortium. Clin Genitourin Cancer 2015;13:e79-85.

20. McGregor BA, McKay RR, Braun DA, et al. Results of a Multicenter Phase II Study of Atezolizumab and Bevacizumab for Patients With Metastatic Renal Cell Carcinoma With Variant Histology and/or Sarcomatoid Features. J Clin Oncol 2020;38:63-70.

21. Rini BI, Motzer RJ, Powles T, et al. Atezolizumab plus Bevacizumab Versus Sunitinib for Patients with Untreated Metastatic Renal Cell Carcinoma and Sarcomatoid Features: A Prespecified Subgroup Analysis of the IMmotion151 Clinical Trial. Eur Urol 2021;27:78-86.

22. Tannir NM, Signoretti S, Choueiri TK, et al. Efficacy and Safety of Nivolumab Plus Ipilimumab versus Sunitinib

Cite this article as: Donskov F. Renal cell carcinoma with non-clear cell histology or sarcomatoid differentiation: recent insight in an unmet clinical need. Ann Transl Med 2021;9(2):97. doi: 10.21037/atm-20-7009 in First-line Treatment of Patients with Advanced Sarcomatoid Renal Cell Carcinoma. Clin Cancer Res 2021;27:78-86.

23. Rini BI, Powles T, Atkins MB, et al. Atezolizumab plus bevacizumab versus sunitinib in patients with previously untreated metastatic renal cell carcinoma (IMmotion 151): a multicentre, open-label, phase 3, randomised controlled trial. Lancet 2019;393:2404-15.

24. Motzer RJ, Escudier B, McDermott DF, et al. Survival outcomes and independent response assessment with nivolumab plus ipilimumab versus sunitinib in patients with advanced renal cell carcinoma: 42-month follow-up of a randomized phase 3 clinical trial. J Immunother Cancer 2020;8:e000891. 